\title{
Sliding thermoelastodynamic instability
}

\author{
By L. Afferrante ${ }^{1}$, M. Ciavarella ${ }^{1, *}$ and J. R. Barber ${ }^{2}$ \\ ${ }^{1}$ CEMEC-PoliBA - Centre of Excellence in Computational Mechanics, \\ V.le Japigia 182, Politecnico di Bari, 70125 Bari, Italy \\ ${ }^{2}$ Department of Mechanical Engineering, University of Michigan, \\ Ann Arbor, MI 48109-2125, USA
}

Numerous mechanisms can give rise to instabilities and vibrations in sliding systems. These can generally be characterized as either elastodynamic (e.g. 'brake squeal') or thermoelastic. The time-scales of these processes differ considerably, so it is usual to neglect coupling between them, i.e. to neglect thermal effects in elastodynamic analyses and to use the quasi-static approximation in thermoelastic analyses. In the present paper, we consider the potential coupling between them in the simplest possible context - a thermoelastodynamic layer sliding against a rigid plane and constrained to one-dimensional displacements. The results show that although the coupling is extremely weak, it has a destabilizing effect on the natural elastodynamic vibration of the layer at arbitrarily low sliding speeds. A numerical solution of the transient equations below the quasi-static critical speed shows that an initial disturbance grows exponentially until periods of separation develop, after which the system approaches asymptotically to a steady state involving periods of contact and separation alternating at the lowest natural frequency of the elastodynamic system. With increasing sliding speed, the proportion of the cycle spent in contact is reduced and the maximum contact pressure increases.

It is important to note that neither a quasi-static thermoelastic analysis, nor an elastodynamic analysis neglecting thermal expansion would predict instability in this speed range. Similar instabilities due to thermoelastodynamic coupling are almost certain to occur in more complex practical sliding systems such as brakes and clutches, implying the need for the incorporation of these effects in commercial analysis software. The proposed mechanism might also provide an explanation of reported experimental observations of vibrations normal to the contact interface during frictional sliding.

Keywords: thermoelastic contact; thermoelastic instability; squeal; frictional vibrations

\section{Introduction}

Instabilities in the sliding of elastic bodies are of interest in a wide range of scientific and industrial applications, including, for example, the sliding of * Author for correspondence (mciava@poliba.it).

The authors wish to dedicate this paper to Professor K. L. Johnson in celebration of his 80th birthday. 
tectonic plates during earthquakes (Ben-Zion 2001; Rice et al. 2001), the mechanism of sliding for rubber-like materials (Barquins et al. 1996), the generation of noise and vibration in automotive brakes (Kincaid et al. 2003) and stick-slip vibrations in machine tool slides and other linear positioning devices (Popp \& Rudolph 2004). These complex phenomena result from the interaction between relatively simple physical processes, notably the elastic deformation of the contacting bodies, the development of frictional forces at the interface opposing the motion and the consequent generation of frictional heat.

Several distinct categories of instability are known to result from these interactions. It has long been known that frictional vibrations can result if the coefficient of friction is a decreasing function of speed or, in the case of stick-slip vibrations, if the static coefficient exceeds the dynamic coefficient. However, Martins et al. (1995) and Adams (1995) have shown that the steady sliding of two elastic half planes can be unstable, even assuming the elementary Coulomb friction law in which the frictional traction is proportional only to the local normal contact pressure. The instability consists of the development of an unstable sinusoidal perturbation in contact pressure which grows exponentially with time as long as full contact and sliding conditions are maintained. This mechanism applies also in more complex geometries and is one explanation of 'squeal' vibrations in automotive brakes (Moirot \& Nguyen 2000). Another category of instability is that associated with the unstable interaction between frictional heating, thermoelastic distortion and contact pressure known as 'thermoelastic instability' or 'TEI' (Barber 1969; Dow \& Burton 1972). In this process, any perturbation in contact pressure causes a corresponding perturbation in heating and hence thermal distortion, which exaggerates the initial perturbation.

Both frictional instabilities and TEI can be analysed using linear perturbation methods, leading to a characteristic equation for the exponential growth rate of an initial perturbation. In the discrete (e.g. finite element) formulation, this takes the form of a linear eigenvalue problem for the growth rate (Yi et al. 2000; Yi \& Barber 2001). However, no one has so far considered the possible interaction between the two mechanisms of instability. Thermoelastic deformations are neglected in the analysis of frictional instabilities and the quasi-static approximation is used in the analysis of TEI. Some justification for this 'decoupling' of the two phenomena is provided by the widely divergent timescales involved. Thermoelastic instabilities occur on the rather slow time-scale of thermal diffusion, whereas elastodynamic processes are governed by the elastic wave speeds in the materials.

In caliper disc brakes, the typical unstable TEI mode involves a set of equally spaced hot spots around the disc and as these pass through the brake pads, they can cause mechanical vibration known as 'hot judder'. However, this is analysed as a 'one-sided' interaction. The TEI problem is assumed to be quasi-static, which then merely serves to define the excitation for a dynamic analysis.

It would clearly be much more satisfactory to develop an analysis of the coupled problem including both elastodynamic and thermoelastic effects in the same perturbation analysis. If the conventional uncoupled wisdom is justified, we should then be able to classify the resulting eigenfunctions into TEI modes and elastodynamic modes. 


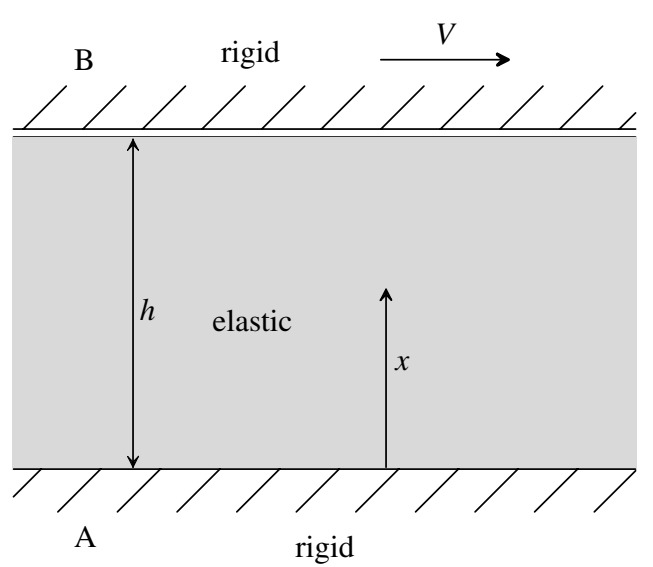

Figure 1. An elastic layer bounded to a rigid body at $x=0$ and sliding against a rigid plane surface at $x=h$.

In the present paper, we initiate this investigation by considering the simplest possible system comprising a one-dimensional elastic layer bonded to a rigid half space and sliding against a second rigid half space. We shall find, surprisingly, that though the modes can in fact be classified in this way, the extremely weak coupling between the two mechanisms destabilizes the elastodynamic natural vibration modes, causing the system to be unstable at arbitrarily low speeds.

\section{Problem statement}

Figure 1 shows an elastic layer $0<x<h$ which is bonded to a stationary rigid plane A at $x=0$. A second rigid body $\mathrm{B}$ moves to the right at velocity $V$ and its plane surface makes sliding contact with the layer at $x=h$. Wear of the sliding bodies is assumed to be negligible. The undeformed thickness of the layer at temperature $T(x)=0$ is assumed to exceed $h$ by a small amount $(\Delta$ so as to ensure an initial contact pressure at the interface, where Coulomb friction conditions are assumed with a coefficient of friction $f$. We also assume that body A is maintained at temperature $T=0$ and that the sliding body $\mathrm{B}$ is a nonconductor, so that all the heat generated by friction flows through the layer. We restrict attention to the one-dimensional plane strain problem, so the only nonzero displacement is $u_{x}$.

These conditions can be summarized in the boundary conditions

$$
\begin{aligned}
& u_{x}=0, \quad T=0, \quad \text { at } x=0, \\
& u_{x}=-\Delta, \quad K \frac{\partial T}{\partial x}=-f V \sigma_{x x}, \quad \text { at } x=h,
\end{aligned}
$$

where $\sigma_{x x}$ is the tensile stress in the layer and $K$ is the thermal conductivity.

The governing equations are the heat conduction equation

$$
\frac{\partial^{2} T}{\partial x^{2}}-\frac{1}{k} \frac{\partial T}{\partial t}=0
$$


the equation of motion

$$
\frac{\partial \sigma_{x x}}{\partial x}-\rho \frac{\partial^{2} u_{x}}{\partial t^{2}}=0
$$

and the one-dimensional plane strain Hooke's law

$$
\sigma_{x x}=\frac{2 \mu(1-\nu)}{(1-2 \nu)} \frac{\partial u_{x}}{\partial x}-\frac{2 \mu(1+\nu) \alpha T}{(1-2 \nu)},
$$

where $\mu, \nu, \rho, k, \alpha$ are, respectively, the modulus of rigidity, Poisson's ratio, density, thermal diffusivity and coefficient of expansion for the material of the layer and $t$ is time.

\section{(a) Dimensionless formulation}

A convenient dimensionless formulation can be developed by defining the quantities

$$
\xi=\frac{x}{h}, \quad \hat{u}=\frac{u_{x}}{h}, \quad \tau=\frac{k t}{h^{2}}, \quad \hat{\sigma}=\frac{(1-2 \nu) \sigma_{x x}}{2 \mu(1-\nu)}, \quad \hat{T}=\frac{\alpha(1+\nu) T}{(1-\nu)},
$$

in which case equations $(2.1)-(2.5)$ reduce to

$$
\begin{gathered}
\hat{u}=0, \quad \hat{T}=0, \quad \text { at } \xi=0, \\
\hat{u}=-\hat{\Delta}, \quad \frac{\partial \hat{T}}{\partial \xi}=-\hat{V} \hat{\sigma}, \quad \text { at } \xi=1, \\
\frac{\partial^{2} \hat{T}}{\partial \xi^{2}}-\frac{\partial \hat{T}}{\partial \tau}=0, \\
\frac{\partial \hat{\sigma}}{\partial \xi}-\gamma^{2} \frac{\partial^{2} \hat{u}}{\partial \tau^{2}}=0, \\
\hat{\sigma}-\frac{\partial \hat{u}}{\partial \xi}+\hat{T}=0,
\end{gathered}
$$

where the dimensionless parameters

$$
\hat{\Delta}=\frac{\Delta}{h}, \quad \hat{V}=\frac{2 \mu \alpha(1+\nu) f V h}{K(1-2 \nu)}, \quad \gamma=\frac{k}{c h}
$$

and

$$
c=\sqrt{\frac{2 \mu(1-\nu)}{\rho(1-2 \nu)}}
$$

is the dilatational wave speed in the layer material. The parameter $\hat{\Delta}$ is the initial compressive strain in the layer and $\hat{V}$ is a dimensionless sliding speed similar in form to that which arises in quasi-static TEI formulations (Joachim-Ajao \& Barber 1998). The parameter $\gamma$ can be interpreted as the ratio between the timescales for elastic wave propagation and for thermal diffusion. The ratio $k / c$ has dimensions of length and for most materials it is extremely small. Some typical values are $1.8 \mathrm{~nm}$ (steel), $23 \mathrm{~nm}$ (copper), $11 \mathrm{~nm}$ (aluminium), $0.8 \mathrm{~nm}$ (aluminium oxide). It follows that $\gamma \ll 1$ unless the layer is extremely thin. 


\section{(b) Steady-state solution}

If a steady state exists, the temperature must be a linear function of $x$ and the stress must be constant. Elementary calculations then show that

$$
\hat{\sigma}=-\frac{2 \hat{\Delta}}{(2-\hat{V})} .
$$

The contact traction must be compressive, so only negative values of $\hat{\sigma}$ are acceptable, showing that a steady state exists if and only if $\hat{V}<2$. For higher sliding speeds, we anticipate that the contact pressure will grow without limit, causing the system to seize (Ciavarella et al. 2003).

\section{(c) Perturbation analysis}

Following Burton et al. (1973) and Barber et al. (1980), we investigate the stability of the steady state by considering the possibility that a small perturbation in the temperature and displacement fields can grow exponentially with time. Thus, we write

$$
\begin{aligned}
& \hat{T}(\xi, \tau)=\hat{T}_{0}(\xi, \tau)+\Theta(\xi) \mathrm{e}^{b \tau}, \\
& \hat{u}(\xi, \tau)=\hat{u}_{0}(\xi, \tau)+U(\xi) \mathrm{e}^{b \tau}, \\
& \hat{\sigma}(\xi, \tau)=\hat{\sigma}_{0}(\xi, \tau)+S(\xi) \mathrm{e}^{b \tau},
\end{aligned}
$$

where $\hat{T}_{0}, \hat{u}_{0}, \hat{\sigma}_{0}$ represent the unperturbed solution, which satisfies $(2.7)-(2.11)$ with appropriate initial conditions. Substituting (2.15)-(2.17) into (2.7)-(2.11), we find that the perturbation must satisfy the equations

$$
\begin{gathered}
\frac{\mathrm{d}^{2} \Theta}{\mathrm{d} \xi^{2}}-b \Theta=0, \\
\frac{\mathrm{d} S}{\mathrm{~d} \xi}-(\gamma b)^{2} U=0, \\
S-\frac{\mathrm{d} U}{\mathrm{~d} \xi}+\Theta=0,
\end{gathered}
$$

with homogeneous boundary conditions

$$
\begin{aligned}
& U=0, \quad \Theta=0, \quad \text { at } \xi=0, \\
& U=0, \quad \frac{\mathrm{d} \Theta}{\mathrm{d} \xi}+\hat{V} S=0, \quad \text { at } \xi=1 .
\end{aligned}
$$

Equation (2.18) with boundary condition $(2.21)_{(i i)}$ has the solution

$$
\Theta=C_{1} \sinh (\xi \sqrt{b})
$$

where $C_{1}$ is an arbitrary constant. We can then use (2.20) and (2.23) to eliminate $S$ in (2.19), obtaining

$$
\frac{\mathrm{d}^{2} U}{\mathrm{~d} \xi^{2}}-(\gamma b)^{2} U=C_{1} \sqrt{b} \cosh (\xi \sqrt{b}) .
$$

The general solution of this equation is

$$
U(\xi)=\frac{C_{1} \cosh (\xi \sqrt{b})}{\sqrt{b}\left(1-\gamma^{2} b\right)}+C_{2} \exp (\gamma b \xi)+C_{3} \exp (-\gamma b \xi) .
$$


The boundary conditions $(2.21)_{(\mathrm{i})}$ and $(2.22)_{(\mathrm{i})}$ can then be used to determine the constants $C_{2}, C_{3}$ in terms of $C_{1}$, giving

$$
C_{2}=-\frac{C_{1}\left[\exp \left(-\gamma z^{2}\right)-\cosh (z)\right]}{2 z\left(1-\gamma^{2} z^{2}\right) \sinh \left(\gamma z^{2}\right)}, \quad C_{3}=\frac{C_{1}\left[\exp \left(\gamma z^{2}\right)-\cosh (z)\right]}{2 z\left(1-\gamma^{2} z^{2}\right) \sinh \left(\gamma z^{2}\right)},
$$

where

$$
z=\sqrt{b}
$$

For the remaining boundary condition $(2.22)_{(\mathrm{ii})}$, we eliminate $S$ using $(2.20)$ and then substitute for $U, \Theta$ from (2.23) and (2.25) with (2.26), to obtain the characteristic equation

$$
\hat{V}=\frac{\left(1-\gamma^{2} z^{2}\right) \cosh (z) \sinh \left(\gamma z^{2}\right)}{\gamma\left[\cosh \left(\gamma z^{2}\right) \cosh (z)-1-\gamma z \sinh (z) \sinh \left(\gamma z^{2}\right)\right]} .
$$

The zeros of (2.28) define the eigenvalues, $b$, at which non-trivial perturbations of the form (2.15)-(2.17) can exist.

\section{(d) Limiting cases}

We have already remarked that in many cases $\gamma \ll 1$, implying that the time required for an elastic wave to traverse the layer is very short compared with the time-scale of the heat conduction process. In such cases, it is conventional to assume that quasi-static conditions can be assumed, equivalent to setting $\gamma=0$. We then obtain

$$
\hat{V}=\frac{z^{2} \cosh (z)}{\cosh (z)-1}
$$

and the stability boundary corresponds to the passage of a real zero through the origin $z=0$ when $\hat{V}=2$.

Another limit of some interest is that in which thermal effects can be neglected, for example if the coefficient of expansion $\alpha \rightarrow 0$, giving $\hat{V}=0$ for all finite sliding speeds. In this case, equation (2.28) reduces to

$$
\left(1-\gamma^{2} z^{2}\right) \cosh (z) \sinh \left(\gamma z^{2}\right)=0
$$

and it has two distinct sets of zeros. The factor $\cosh (z)=0$ when $z=i(2 n+1) \pi / 2$ and $n$ is an integer, corresponding to $b=-(2 n+1)^{2} \pi^{2} / 4$ and hence to exponentially decaying solutions of the heat conduction equation with mixed homogeneous end conditions. However, $\sinh \left(\gamma z^{2}\right)=\sinh (\gamma b)=0$ when $\gamma b=i n \pi$, corresponding to oscillatory solutions which define undamped elastodynamic oscillations of the layer with fixed-fixed end conditions.

\section{(e) Zeros of the characteristic equation}

Equation (2.28) can be written in the symbolic form

$$
\hat{V}=\mathcal{F}(\gamma, \phi, \psi),
$$

where $b=z^{2}=\phi+i \psi$. Thus, $\phi \equiv \operatorname{Re}(b)$ represents the exponential growth rate of the disturbance and $\psi \equiv \operatorname{Im}(b)$ the frequency of the associated oscillation. We are particularly interested in the effect of $\hat{V}$ on $\phi$, since the system is unstable if there exist any zeros with $\phi>0$. The dimensionless sliding speed $\hat{V}$ must be real, 


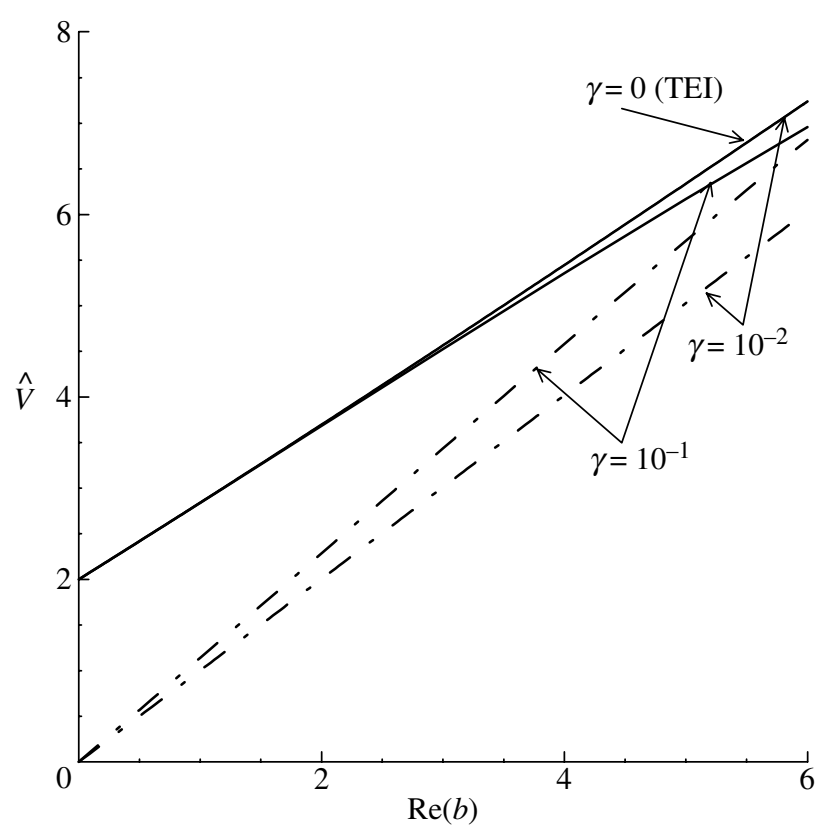

Figure 2. Exponential growth rate $\operatorname{Re}(b)$ as a function of dimensionless sliding speed $\hat{V}$; solid lines, real eigenvalues; dash-dotted lines, complex eigenvalues.

so (2.31) requires that

$$
\begin{aligned}
& \operatorname{Re}(\mathcal{F}(\gamma, \phi, \psi))=\hat{V} \\
& \operatorname{Im}(\mathcal{F}(\gamma, \phi, \psi))=0
\end{aligned}
$$

For a given value of $\gamma$, the zeros of equation (2.28) can be determined parameterically by (i) selecting a value of $\phi$, (ii) solving (2.33) for $\psi$ and then (iii) substituting $\phi, \psi$ into $(2.32)$ to determine $\hat{V}$.

Figure 2 shows the relationship between $\hat{V}$ and $\operatorname{Re}(b)$ for $\gamma=10^{-1}$ and $10^{-2}$. In each case, one real zero and one complex zero is identified. Note that the real zero passes into the complex plane at the value $\hat{V}=2$, exactly as in the limiting case $\gamma=0$. This is to be expected, since a real zero with $\operatorname{Re}(b)=0$ involves no variation of the stress or temperature perturbations $(2.15)-(2.17)$ in time and hence the acceleration terms in the governing equations make no contribution. Thus, the elastodynamic properties of the system have no effect on the stability boundary, if this is determined by a real zero.

However, figure 2 uncovers the surprising conclusion that complex zeros exist with positive real parts for all values of $\hat{V}>0$, showing that the system is unstable for all sliding speeds. In the complex plane, zeros on the imaginary axis representing undamped elastodynamic oscillations of the layer move into the unstable half plane as $\hat{V}$ is increased from zero. This complex root persists for arbitrarily small finite values of $\gamma$, and in fact hardly any further change occurs in the plot of figure 2 for $\gamma<10^{-2}$. 


\section{Numerical study of the transient problem}

The results of $\S 2$ show that arbitrarily small amounts of thermoelastodynamic coupling are sufficient to cause undamped oscillatory states to grow with time. The problem remains linear as long as contact is maintained at the sliding end of the rod, so we must conclude that these perturbations will continue to grow exponentially until they are of sufficient amplitude to cause periods of separation. It is clearly of interest to determine what will be the behaviour of the system in the subsequent nonlinear (or more precisely, piecewise linear) phase of the process. To explore this more fully, the finite-difference method was employed to develop a transient solution for the system. The details of the algorithm are presented in appendix A.

The initial condition was taken to be quiescent with initial temperature everywhere zero, i.e.

$$
\frac{\partial \hat{u}}{\partial \tau}(\xi, 0)=0 ; \quad \hat{T}(\xi, 0)=0 .
$$

It then follows from $(2.8)_{(\mathrm{i})},(2.10)$ and $(2.11)$ that

$$
\hat{\sigma}(\xi, 0)=-\hat{\Delta} \text {. }
$$

The problem so defined is one of 'receding contact' as defined by Dundurs \& Stippes (1970) and extended to thermoelastic contact by Joachim-Ajao \& Barber (1998). It follows that all the physical quantities are linearly proportional to the only non-zero parameter $\hat{\Delta}$ and that the time history of periods of contact and separation is independent of this parameter. Realistic values of $\hat{\Delta}$ are small, since this represents the initial axial compressive strain in the layer, but in view of the linearity of the system, we select the condition $\hat{\Delta}=1$, since results for other values may then be easily extracted by a linear scaling.

The results are qualitatively different depending on whether $\hat{V}$ is greater or less than the TEI critical speed $\hat{V}_{\text {cr }}=2$. For $\hat{V}>2$, the quasi-static analysis has no steady state, since (2.14) predicts physically unrealistic tensile contact stresses and the perturbation analysis predicts a contact pressure that grows monotonically without limit. This phenomenon has been reported before in quasi-static TEI systems (Heckmann \& Burton 1979; Ciavarella et al. 2003) and is known as 'thermoelastic seizure'. For the elastodynamic solution in this speed range, two perturbation modes are unstable, one corresponding to a real growth rate and one to a complex growth rate, as shown in figure 2 . The transient response therefore exhibits exponentially growing oscillations superposed on monotonic growth and the detailed behaviour depends on the relative magnitude of these terms. Figure 3 shows the evolution of the dimensionless contact pressure $\hat{p}(\tau)=-\hat{\sigma}(1, \tau)$ as a function of $\tau$, for $\gamma=10^{-1}$ and $\hat{V}=5,10$. For $\hat{V}=10$, the monotonic growth (real root) dominates and the contact pressure increases without limit without loss of contact, though the oscillation can be seen clearly in figure 3 . However, for $\hat{V}=5$, the amplitude of the oscillatory term grows sufficiently to cause $\hat{p}$ to fall to zero and the subsequent behaviour involves alternating periods of contact and separation. In this phase, contact is governed by the classical unilateral contact conditions of non-negative gap and nonnegative contact pressure. No special treatment is required for the impact condition at the end of a separation period, since the resulting discontinuity of local velocity leads only to a corresponding discontinuity in contact pressure, not 


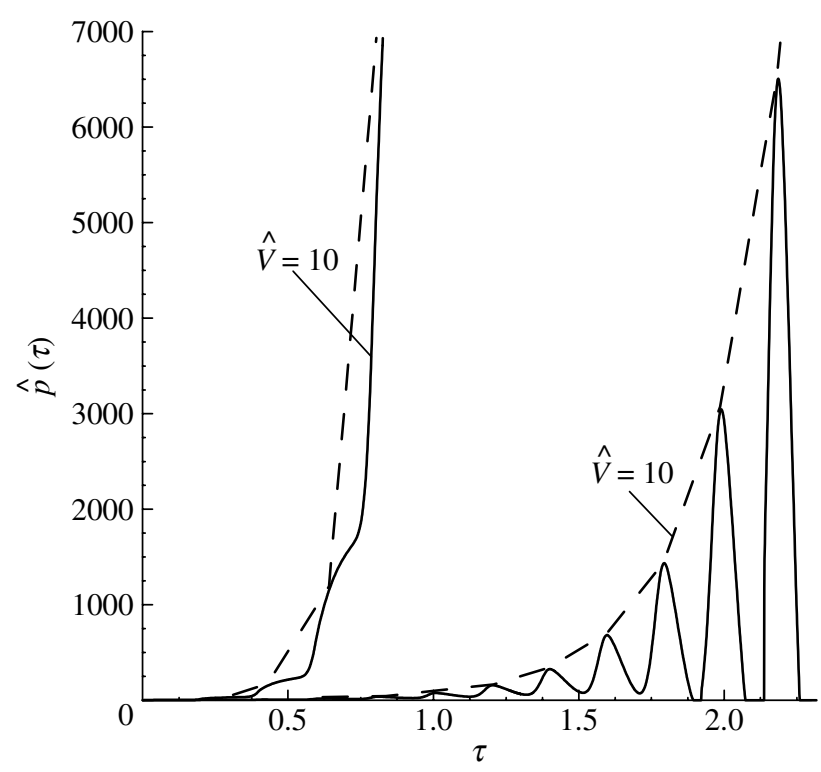

Figure 3. Evolution of the dimensionless contact pressure $\hat{p}$ for $\gamma=10^{-1}$ and $\hat{V}=5,10$.

to a singularity. The long time behaviour for $\hat{V}=5$ involves a series of impacts in which both the maximum contact pressure and the time-averaged mean pressure increase without limit, leading to a state of 'oscillatory seizure'.

More interesting from an engineering perspective are speeds in the range $0<\hat{V}<2$, in which the quasi-static solution predicts a monotonic transition to the steady state (2.14), but the elastodynamic solution predicts unstable growing oscillations. Figure 4 shows the elastodynamic evolution of the dimensionless contact pressure for $\gamma=10^{-1}$ and $\hat{V}=1$. The transient results show an initial trend similar to the quasi-static prediction, but also have a superposed oscillation whose amplitude grows exponentially with time. Eventually, the contact pressure falls to zero and the system undergoes alternating periods of contact and separation, as shown in figure $4 a$.

In this speed range, separation has a stabilizing effect on the process, which then tends asymptotically to a limit cycle. Figure $4 b$ shows the envelope of the maximum pressure reached in each cycle (and is a continuation of the dotted line indicated in figure $4 a$ to larger values of $\tau$ ).

Figure 5 shows three cycles of the fully established limit cycle for $\hat{V}=1$. Note that the contact pressure exhibits a rapid (but not discontinuous) rise at the beginning of the impact and a slower decay. The period between successive impacts was found to be independent of $\hat{V}$ and given by $\tau_{0}=0.2$ for $\gamma=10^{-1}$. This is of course to be expected, since it represents the time taken for a pressure wave to propagate across the layer and be reflected back to the interface. This criterion leads to the more general result $\tau_{0}=2 \gamma$.

\section{(a) Effect of speed}

Similar results were obtained for other speeds in the range $\hat{V}<2$, where the quasi-static solution is stable. As the sliding speed is increased, the maximum contact pressure $p_{\max }$ during the contact phase of the limit cycle increases 

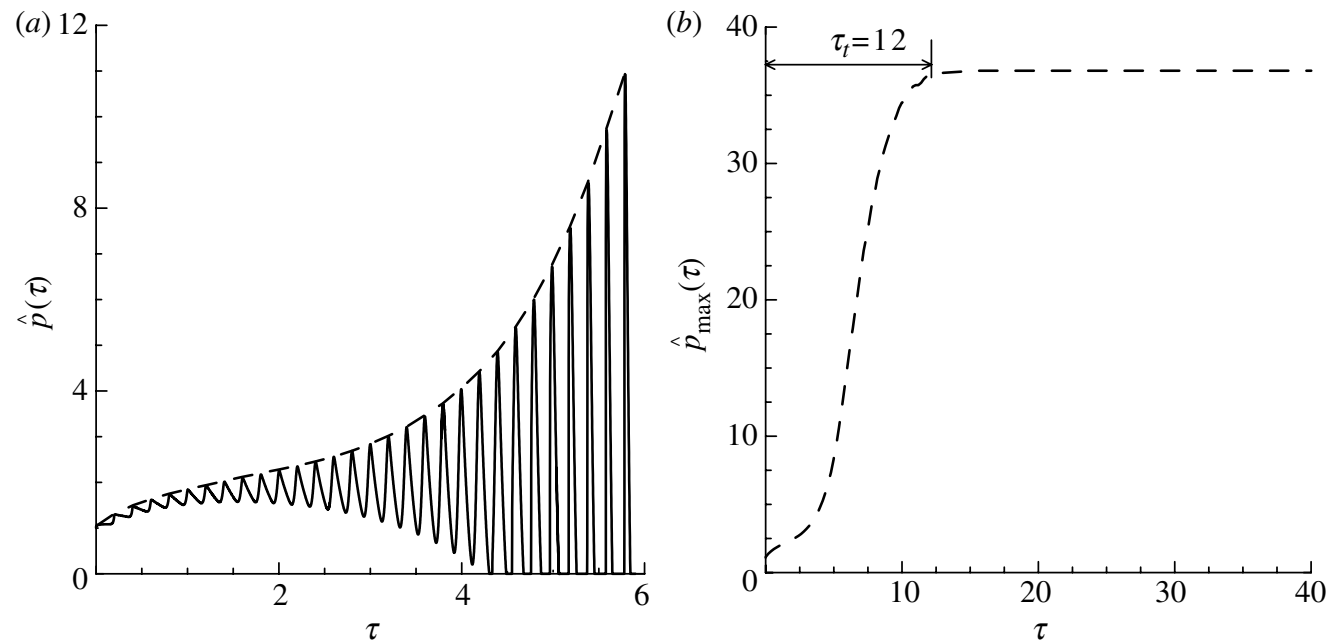

Figure 4. (a) Evolution of the dimensionless contact pressure $\hat{p}$ for $\hat{V}=1, \gamma=10^{-1}$ at small values of dimensionless time $\tau$ and $(b)$ continuation of the envelope of maximum contact pressures $p_{\max }$ at larger values of $\tau$.

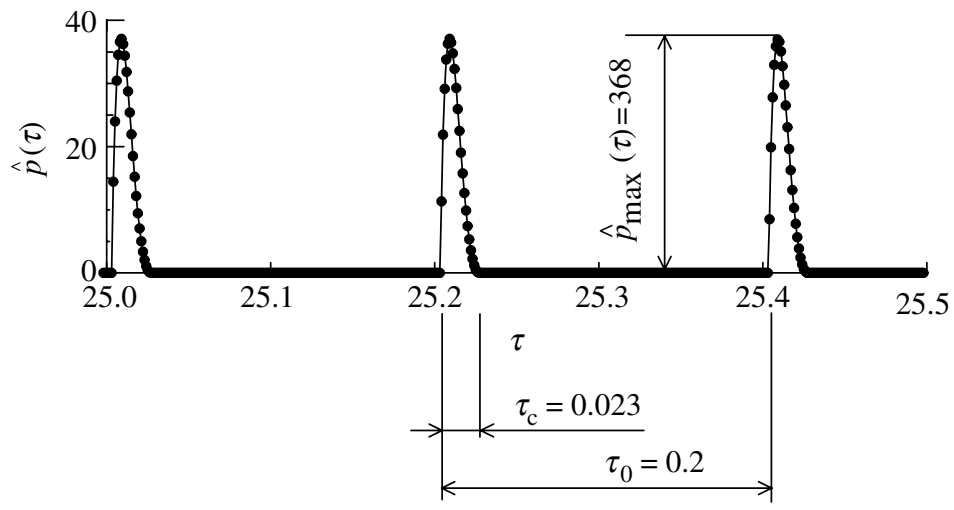

Figure 5. The limit cycle for $\hat{p}$ for $\hat{V}=1, \gamma=10^{-1}$.

substantially, as shown in figure $6 a$. However, the contact period $t_{\mathrm{c}}$ decreases, so the average contact pressure over the contact/separation cycle increases much more slowly, as shown in figure $6 b$. For comparison, we also show in this figure the steady-state contact pressure predicted by the quasi-static analysis (2.14). The two curves are quite close until we approach the quasi-static critical speed, implying that the limit cycle represents a nonlinear oscillation about the quasistatic solution.

Figure $7 a$ shows the length of the initial transient period $\tau_{t}$, defined as the time required for the maximum contact pressure to read $99 \%$ of its steady-state value. For comparison, we also show the time it would take to reach this value if the initial exponential growth were sustained throughout the process. This value,

$$
\tau_{1}=\ln \left(\frac{\hat{p}_{\max }}{\hat{p}(0)}\right) \operatorname{Re}(b),
$$



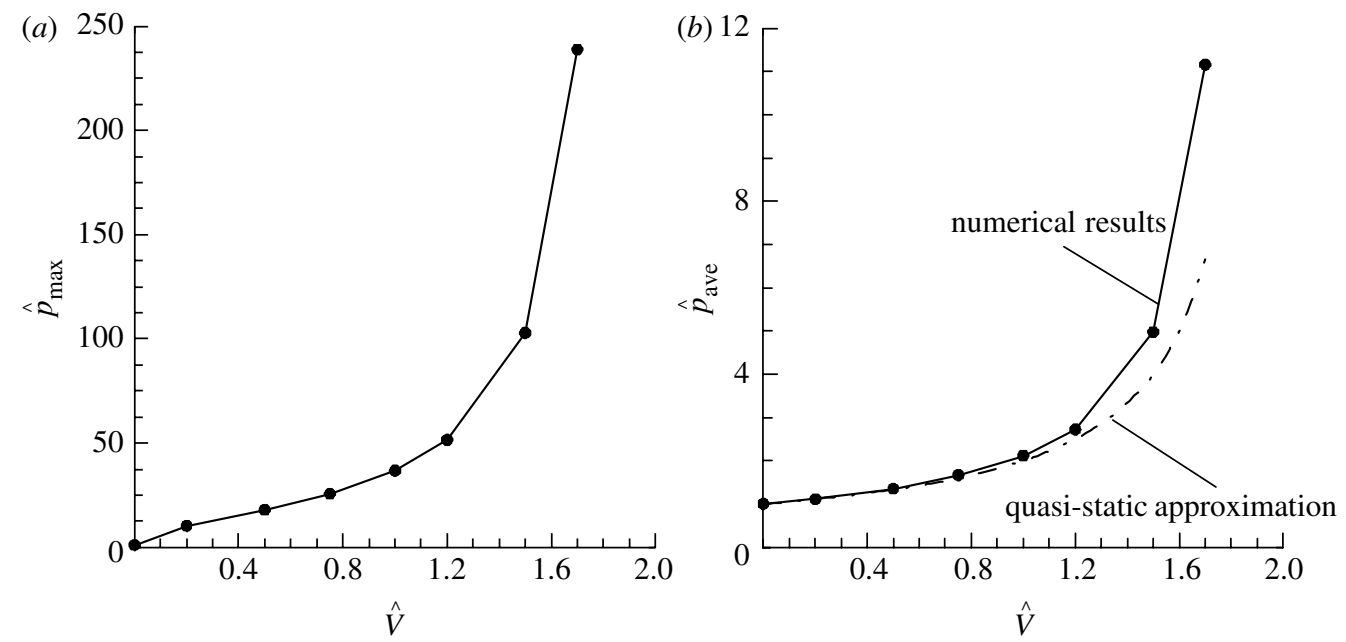

Figure 6. Effect of dimensionless sliding speed $\hat{V}$ for $\gamma=10^{-1}$ on $(a)$ the maximum contact pressure $\hat{p}_{\max }$ during the contact phase and $(b)$ the contact pressure $\hat{p}_{\text {ave }}$ averaged over the period $\tau_{0}$.

is typically about $0.3 \tau_{t}$ as defined above. Also, in figure $7 b$, we show the duration of the contact period $\tau_{\text {c }}$ in the steady state as a function of $\hat{V}$.

After each contact period, a predominantly compressive wave propagates across the layer and is reflected at the fixed support without change in form. However, during the separation period, heat flows out of the layer at the support $\xi=0$, but there is no corresponding heat input at the sliding interface. The corresponding thermoelastic contraction implies that by the time the reflected wave has just reached the sliding end, there is a non-zero gap and hence the first part of this wave will reflect from the traction-free end as a tensile wave until sufficient motion occurs to close the gap. Figure 8 shows the form of the pressure wave in the steady state for $\hat{V}=1$ and a small tensile precursor region is clearly visible.

\section{Discussion}

The results of this simple example demonstrate that a form of thermoelastodynamic instability can occur at arbitrarily small speeds and, in particular, below the quasi-static TEI critical speed. In fact, although the instability mechanism depends on thermal expansion, the resulting vibration has more in common with the phenomenon of brake squeal, since the thermomechanical coupling here destabilizes the natural modes of the dynamic system, rather than establishing a new modal set, as in conventional TEI analyses. It is therefore tempting to refer to this phenomenon as 'hot squeal'.

This mechanism of instability has not been identified previously in the literature and it immediately prompts the question as to whether its effects might be experimentally observable. Anyone who has conducted experiments on systems involving sliding friction can attest to the fact that they are almost invariable noisy (Duffour \& Woodhouse 2004), but many competing models have been proposed to account for this vibration, notably the reduction of friction 

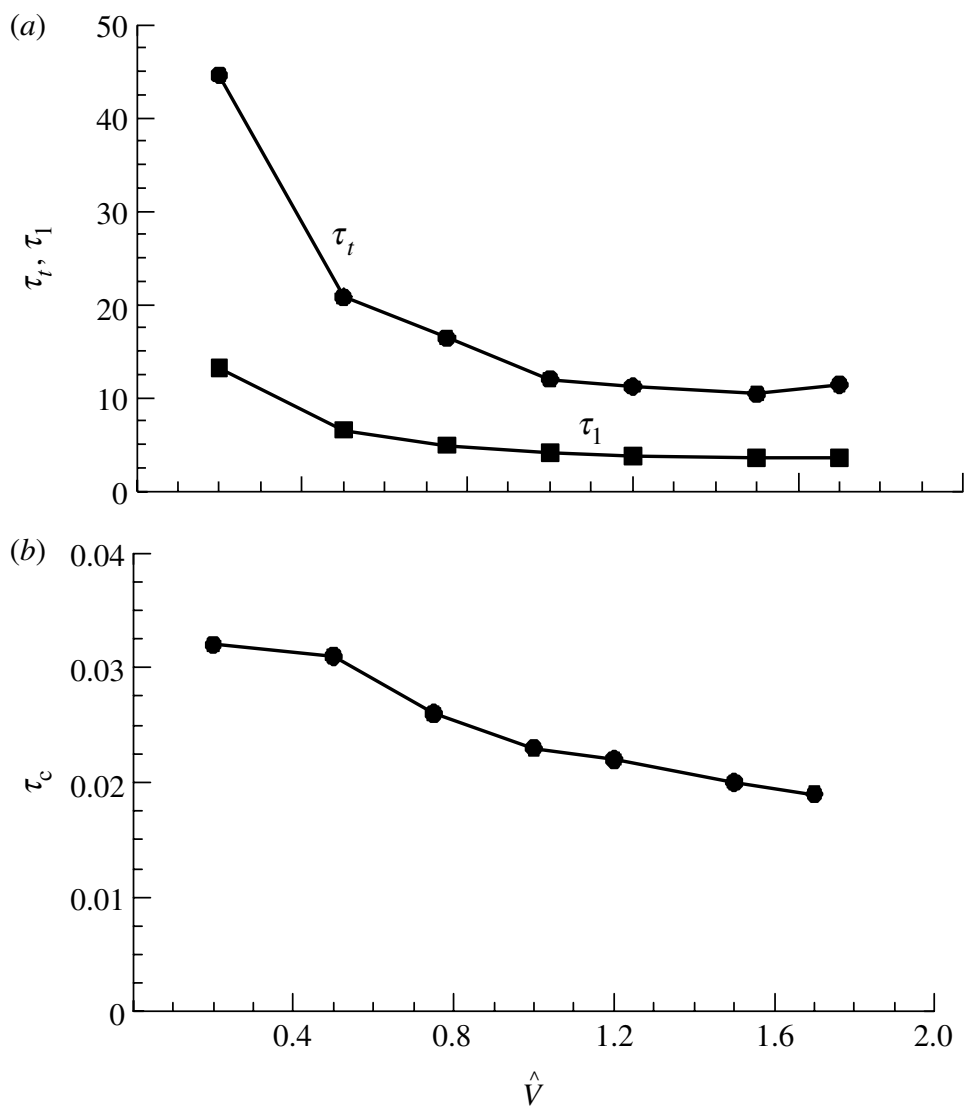

Figure 7. Effect of dimensionless sliding speed $\hat{V}$ for $\gamma=10^{-1}$ on $(a)$ the time taken to reach $99 \%$ of the steady-state maximum contact pressure and $\tau_{1}$ as defined by equation (3.3); $(b)$ the duration of the contact period $\tau_{\mathrm{c}}$.

coefficient with increasing sliding speed (Johnson 2001), the 'sprag' effect (Spurr 1961) and mode coupling due to structural anisotropy and the three-dimensional Coulomb friction law (Moirot \& Nguyen 2000). We should emphasize that none of these effects are active in the present idealized model, which would be stable at all speeds in the absence of thermal expansion.

A common feature observed in most experimental investigations of frictional vibrations is motion of the system in the direction normal as well as tangential to the sliding interface. This effect was first emphasized by Tolstoi (1967), who attributed it to the dynamic effect of sliding over a rough surface. More recently Martins et al. (1990) demonstrated theoretically that normal vibrations can cause frictional (tangential) vibrations, but they were unable to propose a justification for the occurrence and persistence of the normal vibration, on the grounds that an arbitrarily small amount of normal damping would be sufficient to suppress it. Tolstoi showed experimentally that an adequate level of normal damping alone was sufficient to suppress frictional vibrations and that when this was done, the coefficient of friction ceased to exhibit a decrease with sliding speed (at least in the slow speed range of his experiments). 


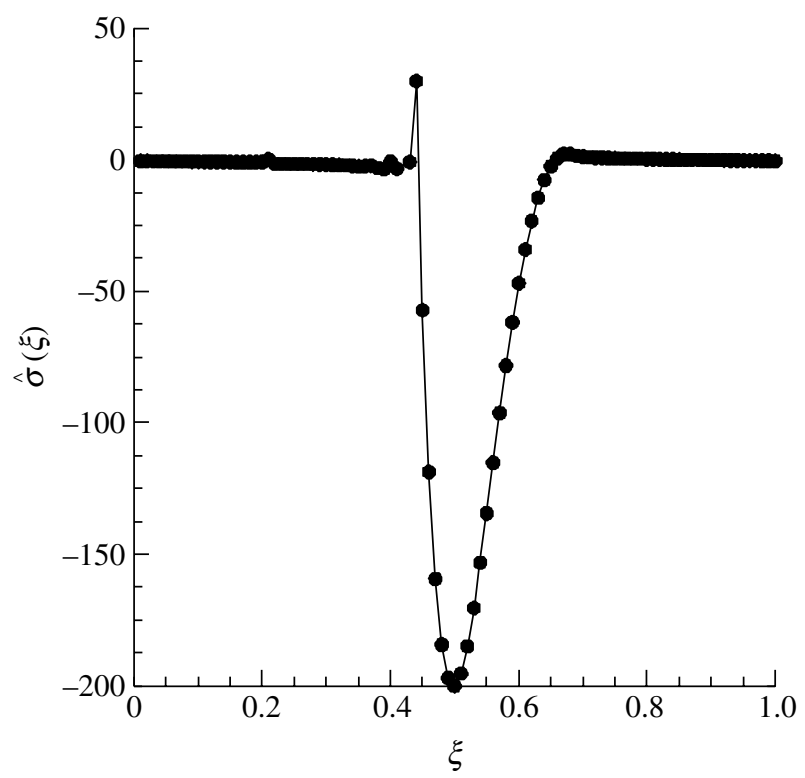

Figure 8. Form of the compression wave in the steady state for $\hat{V}=1, \gamma=10^{-1}$.

The mechanism identified in the present paper provides a plausible explanation for the persistence of self-excited normal vibrations and also predicts that the amplitude of these vibrations increases with sliding speed - a result that is consistent with Tolstoi's experimental results. Tolstoi also argues that the mean separation of the surfaces would increase with sliding speed, on the grounds that the impulses at asperity interactions would increase in magnitude and have a component in the normal direction due to the local surface slope. With the present mechanism, no such argument is necessary to explain an increase in mean separation, since it follows immediately as a consequence of an increase in the amplitude of the contact vibration with speed.

\section{Conclusions}

In this paper, we have identified a new and simple mechanism for the occurrence of frictional vibrations, in which thermomechanical coupling destabilizes the lowest mode of natural vibration. This instability mechanism is distinct from that known as TEI, as established, for example, using the HotSPotTER software (Yi \& Barber 2001). The transient behaviour is characterized by a flutter instability at a frequency close to the first natural frequency of the elastodynamic system and it leads ultimately to a limit cycle with alternating periods of contact and separation also at this frequency.

The instability occurs regardless of the wide difference in time-scales of the thermal and elastodynamic processes as characterized by the smallness of the coupling parameter $\gamma$. In fact, the system is predicted to be unstable for arbitrarily small values of $\gamma$.

The mechanism leads to vibrations normal to the sliding interface and provides a plausible explanation for observations of such vibrations. 
Well-established numerical methods exist for the analysis of both squeal and TEI instabilities. The present results provide cogent reasons for updating these methods to include coupling between thermoelastic and elastodynamic effects.

L.A. and M.C. acknowledge funding from the Centro di Eccellenza in Meccanica Computazionale - Centre of Excellence in Computational Mechanics (CEMEC) and the Ph.D. program in Ingegneria Meccanica Mechanical Engineering, both directed by Prof. M. Napolitano, for sponsoring the visit to the University of Michigan, permitting also the completion of the present work. Also, useful discussions about the numerical study with Profs G. Demelio and P. De Palma are acknowledged.

\section{Appendix A}

When the layer is in contact $(\hat{p}>0)$, the transient problem is defined by equations (2.9)-(2.11) with boundary conditions (2.7) and (2.8), while during separation periods (2.8) is replaced by

$$
\hat{\sigma}=0 ; \quad \frac{\partial \hat{T}}{\partial \xi}=0 ; \quad \text { at } \xi=1,
$$

and the transitions between contact and separation are governed by the unilateral inequalities

$$
\begin{aligned}
\hat{p}(\tau) \equiv-\sigma(1, \tau) & \geq 0 \quad & \text { (contact) } \\
u(1, \tau) \leq-\hat{\Delta} & & \text { (separation) } .
\end{aligned}
$$

The layer is discretized into $n$ equal elements of length $\Delta \xi=1 / n$, so that there are $(n-1)$ internal nodes. We also introduce a sequence of times $\tau^{k}$ at equal intervals $\Delta \tau$. Equation (2.9) is discretized using the Crank-Nicolson implicit scheme, which is unconditionally stable. We obtain

$$
-\frac{r}{2} \hat{T}_{i+1}^{k+1}+(1+r) \hat{T}_{i}^{k+1}-\frac{r}{2} \hat{T}_{i-1}^{k+1}=\frac{r}{2} \hat{T}_{i+1}^{k}+(1-r) \hat{T}_{i}^{k}+\frac{r}{2} \hat{T}_{i-1}^{k},
$$

where

$$
r=\frac{\Delta \tau}{\Delta \xi^{2}}=n^{2} \Delta \tau
$$

This scheme makes use of trapezoidal differencing to achieve second-order accuracy with truncation error of $O\left[(\Delta \tau)^{2}, \Delta \xi^{2}\right]$. However, a tridiagonal system of linear algebraic equations must be solved at each new time-step and for this purpose the Thomas algorithm (Thomas 1949) is used.

When the layer is in contact, boundary condition $(2.8)_{(\mathrm{ii})}$ can be discretized as

$$
\left(\hat{T}_{n}^{k+1}-\hat{T}_{n-1}^{k+1}\right)+\hat{V}\left(\hat{u}_{n}^{k+1}-\hat{u}_{n-1}^{k+1}-\Delta \xi \hat{T}_{n}^{k+1}\right)=0
$$

where we have used (2.11) to eliminate $\hat{\sigma}$. The updating algorithm then requires us to solve the system of algebraic equations

$$
\begin{aligned}
-\frac{r}{2} \hat{T}_{2}^{j+1}+(1+r) \hat{T}_{1}^{j+1} & =C_{1}, \\
-\frac{r}{2} \hat{T}_{i+1}^{j+1}+(1+r) \hat{T}_{i}^{j+1}-\frac{r}{2} \hat{T}_{i-1}^{j+1} & =C_{i}, \quad i=2, \ldots, n-2, \\
\left(1+r-\frac{r / 2}{1-\hat{V} \Delta \xi}\right) \hat{T}_{n-1}^{j+1}-\frac{r}{2} \hat{T}_{n-2}^{j+1} & =C_{n-1},
\end{aligned}
$$


where

$$
\begin{aligned}
C_{1} & =\frac{r}{2} \hat{T}_{2}^{j}+(1-r) \hat{T}_{1}^{j}, \\
C_{i} & =\frac{r}{2} \hat{T}_{i+1}^{j}+(1-r) \hat{T}_{i}^{j}+\frac{r}{2} \hat{T}_{i-1}^{j}, \quad i=2, \ldots, n-2, \\
C_{n-1} & =\frac{r}{2} \hat{T}_{n}^{j}+(1-r) \hat{T}_{n-1}^{j}+\frac{r}{2} \hat{T}_{n-2}^{j}-\frac{r}{2} \frac{\hat{V}\left(\hat{u}_{n}^{j+1}-\hat{u}_{n-1}^{j+1}\right)}{1-\hat{V} \Delta \xi} .
\end{aligned}
$$

During separation periods, the same equations apply, except that the terms involving $\hat{V}$ drop from the boundary condition (2.8) and hence the modified forms of (A 7)-(A 12) can be obtained by setting $\hat{V}=0$.

Eliminating $\hat{\sigma}$ from $(2.10)$, we have

$$
\frac{\partial^{2} \hat{u}}{\partial \xi^{2}}-\gamma^{2} \frac{\partial^{2} \hat{u}}{\partial \tau^{2}}-\frac{\partial \hat{T}}{\partial \xi}=0
$$

This equation is discretized using the Lax-Wendroff scheme (Lax \& Wendroff 1960) for the derivatives of $\hat{u}$, which has a truncation error of $O\left[(\Delta \tau)^{2},(\Delta \xi)^{2}\right]$, and a two-point finite-difference approximation for $\partial \hat{T} / \partial \xi$. It is more convenient to rewrite (A 13) as a system of coupled first-order conservative partial differential equations by defining

from which

$$
\hat{w}=\frac{\partial \hat{u}}{\partial \xi}, \quad \hat{s}=\gamma \frac{\partial \hat{u}}{\partial \tau}
$$

$$
\begin{aligned}
\frac{\partial \hat{w}}{\partial \tau} & =\frac{1}{\gamma} \frac{\partial \hat{s}}{\partial \xi}, \\
\frac{\partial \hat{s}}{\partial \tau} & =\frac{1}{\gamma}\left(\frac{\partial \hat{w}}{\partial \xi}-\frac{\partial \hat{T}}{\partial \xi}\right), \\
\frac{\partial \hat{u}}{\partial \tau} & =\frac{1}{\gamma} \hat{s} .
\end{aligned}
$$

The functions $\hat{w}$ and $\hat{s}$ can then be udpated using the 'one-level' evolution equations

$$
\begin{aligned}
\hat{w}_{i}^{k+1} & =\hat{w}_{i}^{k}+\frac{\beta}{2}\left[\left(\hat{s}_{i+1}^{k}-\hat{s}_{i-1}^{k}\right)+\beta\left(\hat{w}_{i+1}^{k}-2 \hat{w}_{i}^{k}+\hat{w}_{i-1}^{k}\right)\right], \\
\hat{s}_{i}^{k+1} & =\hat{s}_{i}^{k}+\frac{\beta}{2}\left[\left(\hat{w}_{i+1}^{k}-\hat{w}_{i-1}^{k}\right)+\beta\left(\hat{s}_{i+1}^{k}-2 \hat{s}_{i}^{k}+\hat{s}_{i-1}^{k}\right)-\beta\left(\hat{T}_{i+1}^{k}-\hat{T}_{i}^{k}\right)\right],
\end{aligned}
$$

where $\beta=\Delta \tau /(\gamma \Delta \xi)$. This scheme is stable whenever $\beta \leq 1$. However, numerical dissipation errors are found when $\beta$ is less than 1 . For this reason, the numerical simulations were performed for $\beta=1$. Once the value of $\hat{s}_{i}^{k+1}$ has been calculated, the value of $\hat{u}$ can be integrated in time according to equation (A 17), i.e.

$$
\hat{u}_{j}^{k+1}=\hat{u}_{j}^{k}+\frac{\Delta \tau}{\gamma} \hat{s}_{i}^{k+1},
$$

where it should be noted that $\hat{u}_{j}^{k+1}$ has the same truncation error as $\hat{w}_{i}^{k+1}$ and $\hat{s}_{i}^{k+1}$.

In all the examples, the layer was divided into 100 elements of equal length. 


\section{References}

Adams, G. G. 1995 Self-excited oscillations of two elastic half-spaces sliding with a constant coefficient of friction. ASME J. Appl. Mech. 62, 867-872.

Barber, J. R. 1969 Thermoelastic instabilities in the sliding of conforming solids. Proc. R. Soc. A 312, 381-394.

Barber, J. R., Dundurs, J. \& Comninou, M. 1980 Stability considerations in thermoelastic contact. ASME J. Appl. Mech. 47, 871-874.

Barquins, M., Koudine, A. A. \& Vallet, D. 1996 On the existence of Schallamach waves during surface friction in rubber-like material as a function of thickness. Comptes Rendu Mecanique 323, 433-438.

Ben-Zion, Y. 2001 Dynamic ruptures in recent models of earthquake faults. J. Mech. Phys. Solids 49, 2209-2244. (doi:10.1016/S0022-5096(01)00036-9)

Burton, R. A., Nerlikar, V. \& Kilaparti, S. R. 1973 Thermoelastic instability in a seal-like configuration. Wear 24, 177-188. (doi:10.1016/0043-1648(73)90230-5)

Ciavarella, M., Johansson, L., Afferrante, L., Klarbring, A. \& Barber, J. R. 2003 Interaction of thermal contact resistance and frictional heating in thermoelastic instability. Int. J. Solids Struct. 40, 5583-5597. (doi:10.1016/S0020-7683(03)00313-5)

Dow, T. A. \& Burton, R. A. 1972 Thermoelastic instability of sliding contact in the absence of wear. Wear 19, 315-328. (doi:10.1016/0043-1648(72)90123-8)

Duffour, P. \& Woodhouse, J. 2004 Instability of systems with a frictional point contact. Part 1: basic modelling. J. Sound Vib. 271, 365-390. (doi:10.1016/j.jsv.2003.02.002)

Dundurs, J. \& Stippes, M. 1970 Role of elastic constants in certain contact problems. ASME J. Appl. Mech. 37, 965-970.

Heckmann, S. R. \& Burton, R. A. 1979 Seizure of eccentrically loaded journal bearings and sealing rings. ASME J. Lub. Tech. 101, 419-423.

Joachim-Ajao, D. \& Barber, J. R. 1998 Effect of material properties in certain thermoelastic contact problems. ASME J. Appl. Mech. 65, 889-893.

Johnson, K. L. 2001 Dynamic friction. In Tribology research: from model experiment to industrial problem (ed. G. Dalmaz, A. A. Lubrecht, D. Dowson \& M. Priest), pp. 37-45. Amsterdam: Elsevier.

Kinkaid, N. M., O'Reilly, O. M. \& Papaclopoulos, P. 2003 Automotive disc brake squeal. J. Sound Vib. 267, 105-166. (doi:10.1016/S0022-460X(02)01573-0)

Lax, P. D. \& Wendroff, B. 1960 Systems of conservation laws. Commun. Pure Appl. Math. 13, 217-237.

Martins, J. A. C., Oden, J. T. \& Simoes, F. M. F. 1990 A study of static and kinetic friction. Int. J. Eng. Sci. 28, 29-92. (doi:10.1016/0020-7225(90)90014-A)

Martins, J. A. C., Guimaraes, J. \& Faria, L. O. 1995 Dynamic surface solutions in linear elasticity and viscoelasticity with frictional boundary conditions. J. Vib. Acoust. 117, 445-451.

Moirot, F. \& Nguyen, Q. S. 2000 Brake squeal: a problem of flutter instability of the steady sliding solution? Arch. Mech. 52, 645-662.

Popp, K. \& Rudolph, M. 2004 Vibration control to avoid stick-slip motion. J. Vib. Control 10, 1585-1600. (doi:10.1177/1077546304042026)

Rice, J. R., Lapusta, N. \& Ranjith, K. 2001 Rate and state dependent friction and the stability of sliding between elastically deformable solids. J. Mech. Phys. Solids 49, 1865-1898. (doi:10.1016/ S0022-5096(01)00042-4)

Spurr, R. T. 1961 A theory of brake squeal. Proc. Inst. Mech. Engrs (Auto. Div.) 1, 33-52.

Thomas, L. H. 1949 Elliptic problems in linear difference equations over a network. Watson Scientific Computing Laboratory Report. Columbia University, New York.

Tolstoi, D. M. 1967 Significance of the normal degree of freedom and natural normal vibrations in contact friction. Wear 10, 199-213. (doi:10.1016/0043-1648(67)90004-X)

Yi, Y.-B. \& Barber, J. R. 2001 HotSpotter: a finite element software package for evaluating the susceptibility of axisymmetric multidisk brakes and clutches to thermoelastic instability (TEI). http://www-personal.engin.umich.edu/ jbarber/hotspotter.html.

Yi, Y.-B., Barber, J. R. \& Zagrodzki, P. 2000 Eigenvalue solution of thermoelastic instability problems using Fourier reduction. Proc. R. Soc. A 456, 2799-2821. (doi:10.1098/rspa.2000.0641) 